\title{
Multiple displacement amplification for preimplantation genetic diagnosis of fragile $X$ syndrome
}

\author{
H.-S. Lee ${ }^{1}$, M.J. Kim", C.K. Lim ${ }^{1}$, J.W. Cho ${ }^{1}$, I.O. Song ${ }^{2}$ and I.S. Kang ${ }^{2}$ \\ ${ }^{1}$ Laboratory of Reproductive Biology and Infertility, \\ Cheil General Hospital \& Women's Healthcare Center, \\ Kwandong University College of Medicine, Seoul, Korea \\ ${ }^{2}$ Department of Obstetrics and Gynecology, \\ Cheil General Hospital \& Women's Healthcare Center, \\ Kwandong University College of Medicine, Seoul, Korea \\ Corresponding author: H.-S. Lee \\ E-mail: hslee999@gmail.com
}

Genet. Mol. Res. 10 (4): 2851-2859 (2011)

Received January 29, 2011

Accepted May 30, 2011

Published November 17, 2011

DOI http://dx.doi.org/10.4238/2011.November.17.3

\begin{abstract}
Preimplantation genetic diagnosis (PGD) has become an assisted reproductive technique for couples that have genetic risks. Despite the many advantages provided by PGD, there are several problems, including amplification failure, allele drop-out and amplification inefficiency. We evaluated multiple displacement amplification (MDA) for PGD of the fragile $X$ syndrome. Whole genome amplification was performed using MDA. MDA products were subjected to fluorescent PCR of fragile $X$ mental retardation- 1 (FMR1) CGG repeats, amelogenin and two polymorphic markers. In the pre-clinical tests, the amplification rates of the FMRI CGG repeat, DXS1215 and FRAXAC1 were 84.2, 87.5 and 75.0\%, respectively, while the allele dropout rates were $31.3,57.1$ and $50.0 \%$, respectively. In two PGD treatment cycles, 20 embryos among 30 embryos were
\end{abstract}


successfully diagnosed as 10 normal embryos, four mutated embryos and six heterozygous carriers. Three healthy embryos were transferred to the uterus; however, no clinical pregnancy was achieved. Our data indicate that MDA and fluorescent PCR with four loci can be successfully applied to PGD for fragile X syndrome. Advanced methods for amplification of minuscule amounts of DNA could improve the sensitivity and reliability of PGD for complicated single gene disorders.

Key words: Fragile X syndrome; DNA amplification technique; Multiple displacement amplification; Trinucleotide repeat expansion; Preimplantation genetic diagnosis; Allele drop-out

\section{INTRODUCTION}

Preimplantation genetic diagnosis (PGD) for numerical and structural chromosomal abnormalities and single-gene disorders has been successfully applied as an alternative to prenatal diagnosis of inherited diseases. PGD can be used to differentiate between unaffected and affected embryos before embryo transfer in human in vitro fertilization-embryo transfer programs. Therefore, PGD can avoid the initiation of affected pregnancies (Grace et al., 2006; Lee et al., 2007).

Fragile X syndrome (FXS, MIM 309550) is the most common mental retardation disorder. FXS is caused by mutations in the fragile X mental retardation-1 (FMR1) gene located at Xq27.3, more than $95 \%$ of which involve hyperexpansion ( $>200$ CGG repeats) and abnormal hypermethylation of a polymorphic CGG trinucleotide repeat in the 5' untranslated region of the FMR1 gene (Oostra and Chiurazzi, 2001). Phenotypical characteristics include macroorchidism in males, as well as mild facial features like a long face and large protruding ears (Hagerman, 1996). The incidence of this syndrome is estimated to be 1 in 4000 males and 1 in 6000 females based on molecular studies (Turner et al., 1996; de Vries et al., 1997).

Advances in molecular biological techniques allow the application of specific and highly sensitive strategies, such as nested polymerase chain reaction (PCR), multiplex PCR, fluorescent PCR, and whole genome amplification (WGA) for single-cell analysis for PGD. These techniques have been applied to select unaffected embryos for FXS (Sermon et al., 1999; Apessos et al., 2001). However, direct detection of a non-expanded CGG repeat allele or indirect detection using linked polymorphic markers was difficult because of the $\mathrm{G}+\mathrm{C}$-rich sequence and the heterozygosity of the repeats and surrounding sequence in the FMR1 gene, as well as the non-informativity and allele drop-out (ADO) of the linked polymorphic markers. Nevertheless, these direct and indirect detection methods are usually used in the PGD for FXS.

Protocols for PGD of single cells are based on DNA amplification by PCR, which is sufficiently sensitive to detect genetic or genomic variations. Although PCR-based PGD cases are currently performed in all PGD centers, several difficulties associated with singlecell DNA amplification have become evident. These include total amplification failure, ADO, inefficiency of microsatellite amplification, and a limited number of markers for PCR from a single blastomere. Therefore, during the past decade, several WGA techniques, such as primer extension preamplification (Zhang et al., 1992) and degenerate oligonucleotide primed-PCR (Telenius et al., 1992), have been developed and successfully applied to the clinical field, es- 
pecially in PGD, prenatal diagnosis, and forensic medicine. Recently, another WGA method called multiple displacement amplification (MDA) was developed (Dean et al., 2002). Bacteriophage Phi29 DNA polymerase and random exonuclease-resistant hexamers are used to yield large amounts of DNA and consistent DNA amplification regardless of the amount of starting DNA template. This MDA method is also more accurate than the PCR-based WGA methods previously used (Dean et al., 2002). Because of these advantages, MDA is also used in PGD (Hellani et al., 2005; Lledo et al., 2007).

We have developed a PGD strategy for FXS using MDA as a pre-diagnostic step for WGA. Using the MDA product as a template, four separate fluorescent PCRs to amplify the non-expanded CGG repeats and 2 polymorphic markers linked to FMR1 and the amelogenin gene, which allows gender to be determined, were carried out in two consecutive clinical PGD programs for FXS.

\section{MATERIAL AND METHODS}

\section{Patient description}

The patient was a 27-year-old woman carrying a pre-mutation (28 repeats and over 100 repeats of CGG). Her husband showed a number of CGG repeats within the normal range (31 CGG repeats). This couple had experienced a termination of pregnancy for an FXS-affected fetus (31 CGG repeats and a full mutation) that was detected by amniocentesis. As a result of polymorphic marker screening, the DXS1215 (Gyapay et al., 1994) and FRAXAC1 (Richards et al., 1991) polymorphic markers were identified as semi-informative markers in this couple (Figure 1). This couple submitted informed consent before the PGD cycles were started. Ethical approval was obtained from the Cheil General Hospital Ethics Committee before initiating the study.

\section{Isolation and preparation of single lymphocytes}

Lymphocytes were isolated from peripheral blood collected from the heterozygous female partner, her normal male partner, and a normal female control who was heterozygous for the FMR1 CGG repeat, DXS1215 and FRAXAC1 using Ficoll-Paque density gradient separation (Ficoll-Paque ${ }^{\mathrm{TM}}$ PLUS, Amersham Biosciences AB, Uppsala, Sweden) according to the manufacturer protocol. The cell layer containing lymphocytes was removed and diluted with sterile phosphate-buffered saline to a suitable cell density for single-cell isolation. Lymphocytes were then handled with a mouth-controlled fine heat-polished glass micropipette. The lymphocytes were selected and retrieved individually under visual control through an inverted microscope. Each single lymphocyte was loaded into $0.2-\mathrm{mL}$ thin wall PCR tubes containing $1.5 \mu \mathrm{L}$ lysis buffer ( $200 \mathrm{mM} \mathrm{KOH}$ and $50 \mathrm{mM}$ dithiothreitol). The samples were stored at $-70^{\circ} \mathrm{C}$ before analysis.

\section{In vitro fertilization and embryo biopsy procedure}

Ovarian stimulation was done as previously described (Kyu et al., 2004). Following ovarian stimulation, follicles were aspirated and fertilized by intracytoplasmic sperm injec- 
tion. Fertilized zygotes were cultured separately in G-1 medium version 3 (Vitrolife Sweden $\mathrm{AB}$, Kungsbacka, Sweden) for 3 days at $37^{\circ} \mathrm{C}$ in an atmosphere of $5 \% \mathrm{CO}_{2}$. On the third day of culture, embryos were biopsied in $\mathrm{Ca}^{2+} / \mathrm{Mg}^{2+}$-free medium (Biopsy medium; Medicult, Jyllinge, Denmark). Acid Tyrode's solution (Medicult) was applied to create a small hole in the zona pellucida. Biopsy was performed by gentle aspiration using a polished micropipette. One or 2 blastomeres among 6 to 9 cells were biopsied using a single pipette with an inner diameter of $30 \mu \mathrm{M}$. After the blastomere biopsy procedure, the embryos were then repeatedly washed, transferred to G-2 medium version 3 (Vitrolife Sweden $\mathrm{AB}$ ), and cultured at $37^{\circ} \mathrm{C}$ with $5 \% \mathrm{CO}_{2}$ in air. Each blastomere was washed twice with 2 drops G-2 medium version 3 and transferred to sterile $0.2-\mathrm{mL}$ PCR tubes containing $1.5 \mu \mathrm{L}$ alkaline lysis buffer. For each embryo biopsied, blank negative controls were prepared from the wash drops. The embryos were cultured under standard culture conditions until the diagnosis was accomplished. The embryos with normal genotypes were selected and transferred on the fourth day of culture.

\section{Alkaline lysis and DNA extraction procedure}

The single lymphocytes and biopsied blastomeres stored with alkaline lysis buffer were incubated at $65^{\circ} \mathrm{C}$ for $10 \mathrm{~min}$. Then, the alkaline lysis buffer was neutralized by the addition of $1.5 \mu$ L Stop Solution (in REPLI-g ${ }^{\circledR}$ UltraFast Mini Kit; QIAGEN GmbH, Hilden, Germany) before proceeding to MDA.

\section{Multiple displacement amplification}

Denatured cell lysates were used directly for MDA. The isothermal MDA method was performed using bacteriophage Phi29 DNA polymerase, exonuclease-resistant phosphorothioate-modified random hexamer primers and reaction buffer according to the manufacturer instruction (REPLI-g ${ }^{\circledR}$ UltraFast Mini Kit; QIAGEN GmbH) in a $20-\mu$ L reaction volume for at least $2 \mathrm{~h}$ at $30^{\circ} \mathrm{C}$. The reaction was finished by incubation at $65^{\circ} \mathrm{C}$ for $3 \mathrm{~min}$ to inactivate the Phi2 9 polymerase, and DNA amplified using the MDA method was stored at $-20^{\circ} \mathrm{C}$ until required.

\section{Direct analysis of FMR1 CGG repeats and indirect analysis of linked polymorphic markers}

After inactivation of the Phi29 polymerase, the MDA product was diluted to 1:10 with distilled water. Fluorescent PCRs were prepared for 4 separate analyses. The first reaction for direct analysis of CGG repeats was carried out with the diluted MDA product, using FMR1 CGG-specific primer pairs. The total reaction volume was $20 \mu \mathrm{L}$ and contained $3 \mu \mathrm{L}$ of the diluted MDA product, $2 \mu \mathrm{L} \mathrm{10X} \mathrm{PCR} \mathrm{buffer}(500 \mathrm{mM} \mathrm{KCl}, 100 \mathrm{mM}$ Tris-HCl, $\mathrm{pH}$ 8.3), 5\% DMSO, $200 \mathrm{mM}$ dATP, dTTP, dCTP, and 7-deaza-dGTP (Roche Diagnostics GmbH, Mannheim, Germany), 1 IU Neotherm DNA Polymerase (GeneCraft Co., Munster, Germany) and 2 pmol primer (Table 1). The thermal cycling conditions were a $96^{\circ} \mathrm{C}$ denaturation step for $5 \mathrm{~min}$ followed by 15 cycles consisting of $96^{\circ} \mathrm{C}$ for $30 \mathrm{~s}, 67^{\circ} \mathrm{C}$ for $1 \mathrm{~min}$, and $72^{\circ} \mathrm{C}$ for 3 min, and then 25 cycles consisting of $95^{\circ} \mathrm{C}$ for $30 \mathrm{~s}$ with an auto-increment of $0.1^{\circ} \mathrm{C}$ per cycle, $67^{\circ} \mathrm{C}$ for $1 \mathrm{~min}$ and $72^{\circ} \mathrm{C}$ for $3 \mathrm{~min}$, followed by a final extension step of $20 \mathrm{~min}$ at $72^{\circ} \mathrm{C}$ on a GeneAmp PCR System 2700 (Applied Biosystems, Foster City, CA, USA). 


\begin{tabular}{|c|c|}
\hline & Sequences of primers $\left(5^{\prime} \rightarrow 3^{\prime}\right)$ \\
\hline \multicolumn{2}{|l|}{ FMRI CGG repeats } \\
\hline FMRI-forward(outer) & ACGTGACGTGGTTTCAGTGTTTAC \\
\hline FMR1-forward (inner) & FAM-GCTCAGCTCCGTTTCGGTTTCACTTCCGGT \\
\hline FMRI-reverse & AGCCCCGCACTTCCACCACCAGCTCCTCCA \\
\hline \multicolumn{2}{|r|}{ 1 } \\
\hline DXS1215-forward (outer) & ССТTCСТTТССТTGACTCTACACCA \\
\hline DXS1215-forward (inner) & NED-GCCCACTATGTGCCAGTAGGTATG \\
\hline DXS1215-reverse & GGCAAAACATTAAACCTCTCTCATCC \\
\hline \multicolumn{2}{|l|}{ FRAXAC1 } \\
\hline FRAXAC1-forward & FAM-TGGAGACTTCAACACCTCTCTG \\
\hline FRAXAC1-reverse & TGATGAGAGTCACTTGAAGCTG \\
\hline \multicolumn{2}{|l|}{ Amelogenin } \\
\hline Amelogenin-forward (outer) & TGGGCACCCTGGTTATATCAACT \\
\hline Amelogenin-forward (inner) & FAM-CCCTGGGCTCTGTAAAGAATAGTG \\
\hline Amelogenin-reverse & AGGCCAACCATCAGAGCTTAAACT \\
\hline
\end{tabular}

$\overline{F M R 1}=$ fragile $X$ mental retardation -1 ; outer $=$ primers used for the first 'outer' PCRs without multiple displacement amplification.

The second reactions for linkage analysis and gender selection were performed by 3 separate PCRs with primers specific for DXS1215- and FRAXAC1-linked polymorphic markers and the amelogenin gene for gender selection. For each PCR, $1 \mu \mathrm{L}$ of the diluted MDA product, $200 \mathrm{mM}$ of each dNTP (Roche Diagnostics GmbH), 1 IU Neotherm DNA Polymerase (GeneCraft Co), $2 \mu \mathrm{L}$ 10X PCR buffer with $25 \mathrm{mM} \mathrm{MgCl}_{2}$, and 2 pmol of each specific primer pair (forward primers were FAM- or NED-labeled; Table 1) were added to each tube. The reaction was conducted in a total volume of $20 \mu \mathrm{L}$. The thermal cycling profile was as follows: $94^{\circ} \mathrm{C}$ for $2 \mathrm{~min}$ followed by 35 cycles of $95^{\circ} \mathrm{C}$ for $30 \mathrm{~s}, 64^{\circ} \mathrm{C}$ for $30 \mathrm{~s}$ and $72^{\circ} \mathrm{C}$ for 40 s, followed by a final extension step of $10 \mathrm{~min}$ at $72^{\circ} \mathrm{C}$ on a GeneAmp PCR System 2700 . The sequences of the oligonucleotide primers used for PCR are shown in Table 1.

\section{Fragment analysis of the amplified products}

One microliter of the PCR products was added to $9 \mu \mathrm{L}$ genetic analysis grade Hi-Di formamide (Applied Biosystems) and $0.2 \mu \mathrm{L}$ GeneScan-ROX 1000 Size Standard (Applied Biosystems). After boiling for $5 \mathrm{~min}$ at $96^{\circ} \mathrm{C}$, mixtures were capillary-electrophoresed in an ABI 3100 Avant Automatic Genetic Analyzer (Applied Biosystems). Results were analyzed by the GeneScan Analysis version 3.7 software (Applied Biosystems).

\section{RESULTS}

\section{Pre-clinical test using single lymphocytes}

As a result of linkage analysis for this family, the (CA)n microsatellite marker DXS1215 and the intragenic microsatellite marker FRAXAC1 were identified as semi-informative markers (Figure 1). The efficiency and accuracy of our MDA protocol were first evaluated in pre-clinical tests on 19 single lymphocytes collected from the control woman who was heterozygous for the FMR1 CGG repeat, DXS1215 and FRAXAC1. After MDA, four separate reactions were carried out (DXS1215, FRAXAC1, amelogenin, and FMR1 CGG repeats). The 
amplification rate of the FMR1 non-expanded CGG repeats was $84.2 \%(16 / 19)$, and the ADO rate was $31.3 \%(5 / 16)$. For the polymorphic markers DXS1215 and FRAXAC1, amplification rates were 87.5 and $75.0 \%$, while the ADO rates were 57.1 and $50.0 \%$, respectively (Table 2 ).

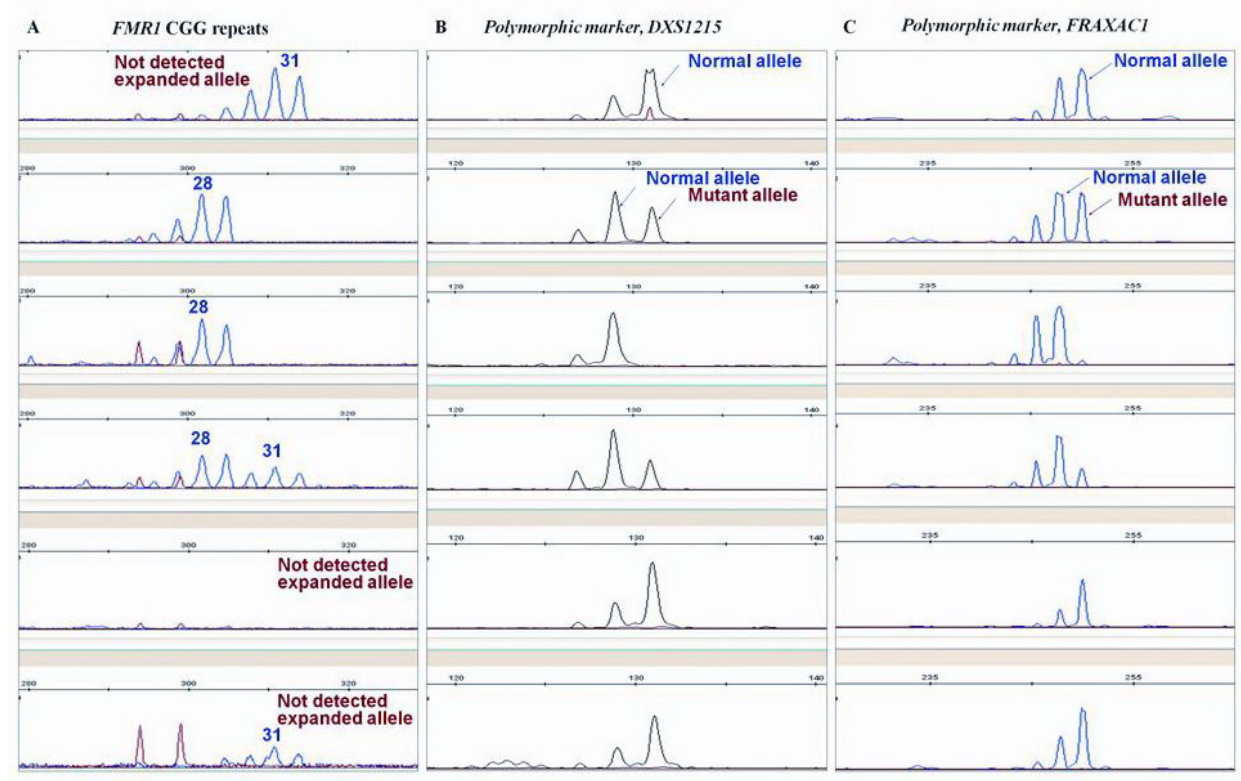

Figure 1. Electropherograms of the non-expanded fragile X mental retardation-1 preimplantation genetic diagnosis (FMR1 CGG) repeats (A) and polymorphic markers, DXS1215 (B) and FRAXAC1 (C) in the second clinical PGD for fragile X syndrome using multiple displacement amplification followed by fluorescent PCR and fragment analysis. Line 1 = normal male partner; line 2 = heterozygous carrier female partner; line 3 = normal male embryo; line 4 = normal female embryo; line 5 = mutant male embryo; line $6=$ heterozygous carrier female embryo. The fully expanded mutant allele (more than 200 repeats) derived from the female partner was not detected (A-1, A-5, and A-6). Red and blue arrows indicate the peaks from the mutant allele and normal allele, respectively. Red peaks in Panel $A$ indicate the GeneScan-ROX 1000 Size Standard.

Table 2. Amplification and allele drop-out rates of pre-clinical tests using single lymphocytes for fragile $\mathrm{X}$ syndrome with or without multiple displacement amplification.

\begin{tabular}{|c|c|c|c|c|}
\hline \multirow[t]{2}{*}{ Applied methods } & \multicolumn{2}{|c|}{ FMRI CGG repeats } & \multirow{2}{*}{$\frac{\text { DXS1215 }}{\text { MDA and fluorescent PCR }}$} & \multirow{2}{*}{$\frac{\text { FRAXAC1 }}{\text { MDA and fluorescent PCR }}$} \\
\hline & $\begin{array}{c}\text { Fluorescent-nested PCR } \\
\text { without MDA }\end{array}$ & MDA and fluorescent PCR & & \\
\hline No. of single lymphocytes & 7 & 19 & 8 & 8 \\
\hline No. of amplified cells & 0 & 16 & 7 & 6 \\
\hline Amplification rates & $0.0 \%$ & $84.2 \%$ & $87.5 \%$ & $75.0 \%$ \\
\hline ADO rates & - & $31.3 \%$ & $57.1 \%$ & $50.0 \%$ \\
\hline
\end{tabular}

FMR1 = fragile $X$ mental retardation- 1 MDA = multiple displacement amplification; $\mathrm{ADO}=$ allele drop-out.

\section{Clinical PGD for fragile $\mathrm{X}$ syndrome}

After the pre-clinical test, the clinical PGD program was applied to a couple who experienced a termination of pregnancy for a fragile X syndrome-affected fetus. In the first 
clinical PGD program, a blastomere was biopsied on embryos with fewer than 6 cells and 2 blastomeres on 6 or more cells. A total of 23 blastomeres were biopsied and diagnosed from 15 embryos on day 3 after fertilization. Of 15 embryos analyzed after pre-amplification using MDA, no PCR product was amplified from 1 embryo (6.7\%) and only amelogenin products were obtained from 3 embryos (20.0\%). Three embryos were diagnosed as unaffected embryos, 1 embryo was identified as mutant, and 3 embryos were carriers. The genetic statuses of four embryos $(26.7 \%)$ were not determined because their results were ambiguous (ADO or incomplete data). Unfortunately, half of the embryos analyzed gave no or incomplete results $(8 / 15,53.3 \%)$. Three healthy embryos were transferred to the mother's uterus on day 4 , resulting in biochemical pregnancy. In the second PGD cycle, a total of 25 blastomeres were biopsied from 15 embryos and analyzed. Four separate reactions for each blastomere were carried out after MDA amplification. Unlike the first cycle, PCR products were amplified and diagnosed from all embryos analyzed. Seven embryos were diagnosed as unaffected embryos, 3 embryos were identified as mutant, and 3 embryos were carriers. Results were not determined in only 2 embryos (13.3\%), because their results were inconclusive (different results for 2 different markers). Of 7 normal embryos, 3 well-developed embryos were transferred to the mother's uterus on day 4. Unfortunately, no pregnancy was achieved (Figure 1 and Table 3).

\begin{tabular}{|c|c|c|c|}
\hline & 1st cycle & 2nd cycle & Total (mean) \\
\hline No. of biopsied embryos & 15 & 15 & $30(15.0)$ \\
\hline No. of diagnosed embryos & 7 & 13 & $20(10.0)$ \\
\hline Successful diagnosis rate & $46.7 \%$ & $86.7 \%$ & $66.7 \%$ \\
\hline No. of unaffected embryos & $3(42.9 \%)$ & $7(53.9 \%)$ & $10(50.0 \%)$ \\
\hline ADO rates (DXS1215/FRAXAC1)* & $50.0 / 40.0 \%$ & $50.0 / 40.0 \%$ & $50.0 / 40.0 \%$ \\
\hline No. of transferred embryos & 3 & 3 & $6(3.0)$ \\
\hline Clinical pregnancy & Biochemical pregnancy & No & \\
\hline
\end{tabular}

*ADO (allele drop-out) rates were calculated only on female normal embryos for polymorphic markers, DXS1215 and FRAXAC1 because semi-informative markers alone were available in this family.

\section{DISCUSSION}

Multiplex (fluorescent) nested PCR methods followed by direct sequencing and/or fragment analysis provided reasonable reliability in single-cell analysis in our center. These protocols have been successfully applied to many clinical PGD cases for couples at high risk of having children with single-gene disorders (Lee et al., 2005, 2006, 2007).

Initially, as for other single-gene disorders, we developed a strategy for FXS using fluorescent nested PCR with genomic DNA followed by fragment analysis without whole genome amplification. However, when we applied this strategy to single lymphocytes in the pre-clinical tests, we did not obtain any results (Table 2). Direct detection of the normal nonexpanded CGG repeat allele as well as the expanded CGG repeat allele is difficult because of the lack of PCR sensitivity and the high $\mathrm{G}+\mathrm{C}$ content of the CGG repeat sequences and the surrounding sequences of the FMR1 gene despite specific PCR protocols using the GCRICH PCR System (Roche Diagnostics GmbH) and Expand Long Template PCR System (Roche Diagnostics GmbH). Therefore, we developed a new PGD strategy for FXS. We used 
MDA as a WGA method because large amounts of high-quality DNA could be obtained. MDA produces enough DNA from a single cell for multiple PCRs. Using the MDA products as a template, we performed only a single round of PCR, which decreases the time required for the PGD program. This strategy is a reliable protocol for obtaining material from a sample with a minuscule amount of DNA, such as a single cell, and several reports have demonstrated high amplification rates (88-100\%) with an ADO rate of 7-31\% when using MDA-amplified DNA as a template (Handyside et al., 2004; Hellani et al., 2004, 2005; Burlet et al., 2006; Lledo et al., 2007). In particular, Burlet et al. (2006) reported successful diagnosis, pregnancy and delivery in the PGD program using MDA for FXS. The amplification rate was increased by this method from 41 to $66 \%$ so that embryos with no results were rarer (14 vs $45 \%$ without MDA).

In our pre-clinical test for FMRI non-expanded CGG repeats and the DXS1215 and FRAXAC1 polymorphic markers, amplification rates were $75-84 \%$ and ADO rates were 31 $57 \%$. Although our data showed lower PCR amplification rates and higher ADO rates than those previously reported, the reliability and the accuracy of the diagnosis were improved by combining both direct detection of the CGG repeat and indirect detection of two polymorphic markers. The use of the non-expanded CGG repeats, two polymorphic markers, and the amelogenin gene for gender selection allowed us to decrease misdiagnosis due to ADO and to increase the successful diagnosis rate. Furthermore, in the case of the clinical PGD program, we have biopsied two blastomeres from embryos with more than 6 blastomeres. Amplification failure or misdiagnosis due to $\mathrm{ADO}$ at one blastomere or one locus was avoided by extrapolating the results obtained from other blastomeres or other loci. Inconclusive or ambiguous results led to no transfer of the embryos. In our cases, a total of 10 embryos $(33.3 \%)$ were diagnosed as normal, and 6 of these embryos were transferred to the uterus. Unfortunately, no clinical pregnancy was achieved.

Our data demonstrate that MDA as a WGA method can produce enough DNA from a single cell for multiple PCR analyses. A PGD strategy using MDA followed by fluorescent PCR may be applied to the diagnosis of many kinds of single-gene disorders. Moreover, by using MDA methods, molecular karyotyping of a single cell such as array comparative genomic hybridization may be used to assess chromosomal abnormalities.

\section{REFERENCES}

Apessos A, Abou-Sleiman PM, Harper JC and Delhanty JD (2001). Preimplantation genetic diagnosis of the fragile X syndrome by use of linked polymorphic markers. Prenat. Diagn. 21: 504-511.

Burlet P, Frydman N, Gigarel N, Kerbrat V, et al. (2006). Multiple displacement amplification improves PGD for fragile X syndrome. Mol. Hum. Reprod. 12: 647-652.

de Vries BB, van den Ouweland AM, Mohkamsing S, Duivenvoorden HJ, et al. (1997). Screening and diagnosis for the fragile X syndrome among the mentally retarded: an epidemiological and psychological survey. Collaborative fragile X study group. Am. J. Hum. Genet. 61: 660-667.

Dean FB, Hosono S, Fang L, Wu X, et al. (2002). Comprehensive human genome amplification using multiple displacement amplification. Proc. Natl. Acad. Sci. U. S. A. 99: 5261-5266.

Grace J, El-Toukhy T, Scriven P, Ogilvie C, et al. (2006). Three hundred and thirty cycles of preimplantation genetic diagnosis for serious genetic disease: clinical considerations affecting outcome. BJOG 113: 1393-1401.

Gyapay G, Morissette J, Vignal A, Dib C, et al. (1994). The 1993-94 genethon human genetic linkage map. Nat. Genet. 7: 246-339.

Hagerman RJ (1996). Physical and Behavioral Phenotype. In: Fragile X Syndrome: Diagnosis, Treatment and Research (Hagerman RJ and Silverman AC, eds.). John Hopkins University Press, Baltimore and London, 3-87.

Handyside AH, Robinson MD, Simpson RJ, Omar MB, et al. (2004). Isothermal whole genome amplification from single 
and small numbers of cells: a new era for preimplantation genetic diagnosis of inherited disease. Mol. Hum. Reprod. 10: 767-772.

Hellani A, Coskun S, Benkhalifa M, Tbakhi A, et al. (2004). Multiple displacement amplification on single cell and possible PGD applications. Mol. Hum. Reprod. 10: 847-852.

Hellani A, Coskun S, Tbakhi A and Al-Hassan S (2005). Clinical application of multiple displacement amplification in preimplantation genetic diagnosis. Reprod. Biomed. Online 10: 376-380.

Kyu LC, Hyun JJ, Mi MD, Lee HS, et al. (2004). Efficacy and clinical outcome of preimplantation genetic diagnosis using FISH for couples of reciprocal and Robertsonian translocations: the Korean experience. Prenat. Diagn. 24: 556-561.

Lee HS, Choi HW, Lim CK, Park SY, et al. (2005). Efficacy of duplex-nested PCR and fluorescent PCR in the preimplantation genetic diagnosis for Duchenne muscular dystrophy. Korean J. Fertil. Steril. 32: 17-26.

Lee HS, Choi HW, Lim CK, Koong MK, et al. (2006). Identification of a novel single nucleotide polymorphism of HADHA gene at a referred primer-binding site during pre-diagnostic tests for preimplantation genetic diagnosis. $J$. Korean Med. Sci. 21: 794-799.

Lee HS, Jun JH, Choi HW, Lim CK, et al. (2007). Preimplantation genetic diagnosis for ornithine transcarbamylase deficiency by simultaneous analysis of duplex-nested PCR and fluorescence in situ hybridization: a case report. $J$. Korean Med. Sci. 22: 572-576.

Lledo B, Bernabeu R, Ten J, Galan FM, et al. (2007). Preimplantation genetic diagnosis of X-linked adrenoleukodystrophy with gender determination using multiple displacement amplification. Fertil. Steril. 88: 1327-1333.

Oostra BA and Chiurazzi P (2001). The fragile X gene and its function. Clin. Genet. 60: 399-408.

Richards RI, Holman K, Kozman H, Kremer E, et al. (1991). Fragile X syndrome: genetic localisation by linkage mapping of two microsatellite repeats FRAXAC1 and FRAXAC2 which immediately flank the fragile site. J. Med. Genet. 28: 818-823.

Sermon K, Seneca S, Vanderfaeillie A, Lissens W, et al. (1999). Preimplantation diagnosis for fragile X syndrome based on the detection of the non-expanded paternal and maternal CGG. Prenat. Diagn. 19: 1223-1230.

Telenius H, Carter NP, Bebb CE, Nordenskjold M, et al. (1992). Degenerate oligonucleotide-primed PCR: general amplification of target DNA by a single degenerate primer. Genomics 13: 718-725.

Turner G, Webb T, Wake S and Robinson H (1996). Prevalence of fragile X syndrome. Am. J. Med. Genet. 64: 196-197.

Zhang L, Cui X, Schmitt K, Hubert R, et al. (1992). Whole genome amplification from a single cell: implications for genetic analysis. Proc. Natl. Acad. Sci. U. S. A. 89: 5847-5851. 